Le Lait (1984), 64, 129-140

\title{
Microfiltration tangentielle avec décolmatage sur membranes céramiques
}

\author{
par \\ S. GALAJ*, A. WICKER*, J.-P. DUMAS* \\ et J. GILLOT**, D. GARCERA**
}

R és u m é

La microfiltration tangentielle avec décolmatage est étudiée sur une membrane céramique tubulaire en alumine pure à structure anisotrope dont la couche filtrante possède des pores de diamètre $0,2 \mu \mathrm{m}$.

Un appareil de microfiltration tangentielle original de très faible volume mort et dénué de boucle de recirculation est présenté. Il permet d'obtenir en tout point de la membrane et rapidement des conditions identiques de pression, vitesse tangentielle et concentration. Le décolmatage est assuré par un organe refoulant une fraction de perméat sous des conditions ajustables.

Un modèle de filtration tangentielle avec décolmatage prenant en compte des interactions mécaniques réversibles au niveau des pores superficiels permet de décrire l'évolution du débit relatif de filtration. Un taux critique de colmatage a été mis en évidence au-delà duquel le décolmatage dévient inefficace.

Des vérifications expérimentales effectuées sur de l'eau et du vin montrent l'intérêt de ce modèle pour la définition des conditions optimales de filtration.

Mots clés :

Microfiltration tangentielle - Décolmatage - Membrane céramique.

* Laboratoires de Marcoussis, Centre de Recherches de la Compagnie Générale d'Electricité, route de Nozay - 91460 Marcoussis.

** Ceraver, usine de Bazet, B.P. 113 - 65001 Tarbes. 


\section{S u m m a r y}

Cross-flow filtration with backflushing the membrane by reversing the permeation direction is studied on a tubular microporous ceramic membrane having pores diameter around $0.2 \mathrm{\alpha m}$.

An experimental set-up with low dead volume and without recirculating system is presented. It allows to obtain, in a short time, the same value for pressure, flow rate and concentration along the membrane.

A theoretical model, based upon reversible mechanical forces taking place on the first layer of the membrane, is developed, leading to the description of the time dependence of permeate flux, clogging ratio and backflush conditions.

Results of experimental assays done on tap-water and crude-wine demonstrate the ability of the model for monitoring filtration operations.

Key words:

Cross-flow filtration - Backflushing - Microporous ceramic membrane.

\section{INTRODUCTION}

Dans le domaine de la microfiltration, où les particules à retenir ont un diamètre moyen compris entre 0,1 et $10 \mu \mathrm{m}$ environ, la technique la plus communément répandue est celle de la filtration frontale dans laquelle il y a formation de gâteau entraînant une diminution progressive du débit du perméat. Pour pallier cet inconvénient, il suffit de faire circuler rapidement la suspension à la surface du filtre : c'est le principe de la filtration tangentielle utilisé depuis plusieurs années dans l'ultrafiltration et dans l'osmose inverse. Malgré la circulation tangentielle, les membranes peuvent se colmater progressivement, c'est pourquoi un décolmatage périodique est effectué par injection de filtrat à contre-courant à travers la membrane. Dans le domaine de l'ultrafiltration, l'étude des mécanismes a accompagné les réalisations pratiques (Lopez-Leiva, 1980) de façon à rechercher les meilleures conditions opératoires. C'est ainsi que des modèles de colmatage (Blatt et coll., 1970 ; Porter, 1972 ; Howell et Vélicangil, 1980 ) et de décolmatage par pression inverse ont été proposés (Belfort, Baltutis et Blatt, 1980).

Le but de cet article est d'appréhender le mécanisme de filtration mis en jeu lors de la filtration tangentielle avec décolmatage sur membrane minérale microporeuse. Le modèle proposé, basé sur les interactions mécaniques existant au niveau des pores superficiels, 
est confronté aux résultats expérimentaux obtenus à l'aide d'un appareil de laboratoire, dénué de boucle de recirculation, utilisant un tube filtrant microporeux en céramique.

\section{APPAREILLAGE}

L'appareillage utilisé se compose principalement d'une membrane filtrante en céramique et d'un système permettant la filtration.

\section{Caractéristiques des membranes minérales}

Structure. La structure de ces membranes minérales est asymétrique : la paroi filtrante est constituée de plusieurs couches superposées. La couche filtrante proprement dite d'une épaisseur comprise entre 15 et $20 \mu \mathrm{m}$ repose sur un support épais de un à plusieurs millimètres dont les pores ont un diamètre moyen compris entre 10 et $15 \mu \mathrm{m}$. Ces couches sont liées entre elles de façon monolithique par des liaisons céramiques formées lors de la fabrication par un procédé de frittage. La figure 1 représente la coupe d'une paroi fil-

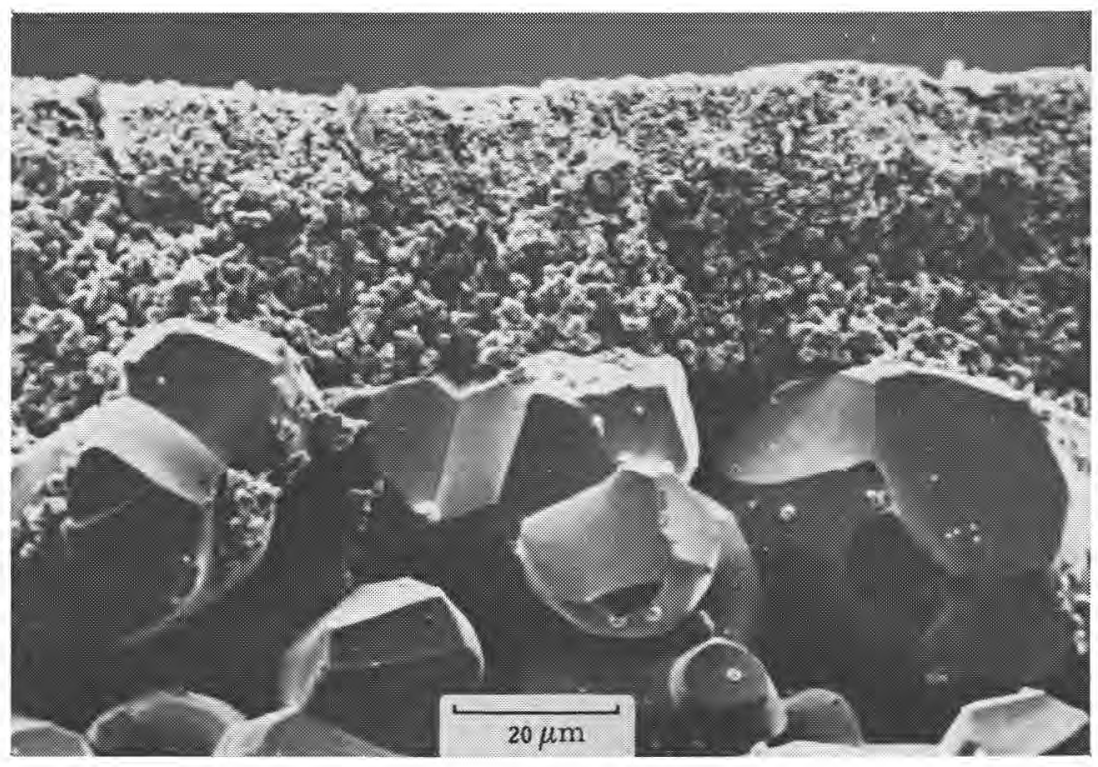

fig. 1

Coupe d'une paroi filtrante à porosité $0,2 \mu \mathrm{m}$.

Cross-section of a $0.2 \mu \mathrm{m}$ membrane. 
trante; on y distingue une partie du support à porosité grossière, une couche intermédiaire épaisse de $20 \mu \mathrm{m}$ environ et la couche filtrante d'une épaisseur de $15 \mu \mathrm{m}$ et de diamètre de pares de 0,2 $\mu \mathrm{m}$.

Propriétés perméamétriques. Selon les conditions de fabrication et la taille des pores considérée, les perméabilités obtenues sont présentées dans le tableau 1.

\section{TABLEAU $1-T A B L E 1$}

Propriétés perméamétriques des membranes minérales

Permeability properties of mineral membranes

\begin{tabular}{|c|c|c|}
\hline $\begin{array}{c}\text { Diamètres moyen } \\
\text { des pores de la couche } \\
\text { la plus fine } \\
\mu \mathrm{m}\end{array}$ & $\begin{array}{l}\text { Perméabilité à l'air } \\
\text { à } 20^{\circ} \mathrm{C} \text { et } 1 \text { atm. } \\
\mathrm{N} \mathrm{m}^{3} \cdot \mathrm{h}^{-1} \cdot \mathrm{m}^{-2} \cdot \mathrm{bar}^{-1}\end{array}$ & $\begin{array}{c}\text { Perméabilité à l'eau } \\
\text { à } 20^{\circ} \mathrm{C} \\
\mathrm{m}^{3} \cdot \mathrm{h}^{-1} \cdot \mathrm{m}^{-2} \cdot \mathrm{bar}^{-1}\end{array}$ \\
\hline $0,2 \begin{array}{l}\text { type } \mathrm{A} \\
\text { type } \mathrm{B}\end{array}$ & $\begin{array}{r}500 \\
1000\end{array}$ & $\begin{array}{r}1,5-2 \\
3-4\end{array}$ \\
\hline 1,5 & 4500 & 40 \\
\hline 10 & 10000 & 120 \\
\hline
\end{tabular}

Composition et aspect des éléments filtrants. Le support ainsi que les couches filtrantes à porosité fine sont constitués d'alumine pure à $99 \%$. Les éléments filtrants sont des tubes de diamètre extérieur allant de 10 à $60 \mathrm{~mm}$, la longueur pouvant atteindre $750 \mathrm{~mm}$. La couche fine est déposée de préférence à l'intérieur du tube. Ces tubes peuvent être assemblés en cartouche (par exemple 19 tubes de $\varnothing 10 \mathrm{~mm}$ pour une longueur de $750 \mathrm{~mm}$ ).

Avantages des membranes minérales asymétriques. Ces membranes combinent de très bonnes propriétés de résistance mécanique, d'inertie chimique et de réfractairité de l'alumine pure à une structure qui réduit au minimum les pertes de charge, ce qui conduit aux caractéristiques suivantes :

- débit élevé pour une pression de filtration donnée; inverses ;

- possibilité d'appliquer des pressions élevées directes ou

- plage de température d'utilisation importante $\left(0\right.$ à $\left.300^{\circ} \mathrm{C}\right)$;

- phénomène de relargage supprimé ;

- utilisation possible de fluides corrosifs. 


\section{Appareillage de filtration}

Description (fig. 2). La membrane céramique est insérée dans une cartouche permettant un écoulement tangentiel circulaire du fluide à filtrer. Un rotor situé à l'intérieur du tube céramique entraîne le fluide. La cartouche remplit plusieurs fonctions : support de la membrane, séparation d'une suspension d'entrée en perméat et rétentat, assurer l'écoulement tangentiel, résister à la pression, à la température et aux agents de nettoyage, et permettre l'échange de la membrane. Les éléments rigides de la cartouche sont en acier inoxydable, la surface utilisable de la membrane céramique est de $180 \mathrm{~cm}^{2}$, le volume de fluide en contact avec la membrane est d'environ $20 \mathrm{~cm}^{3}$.

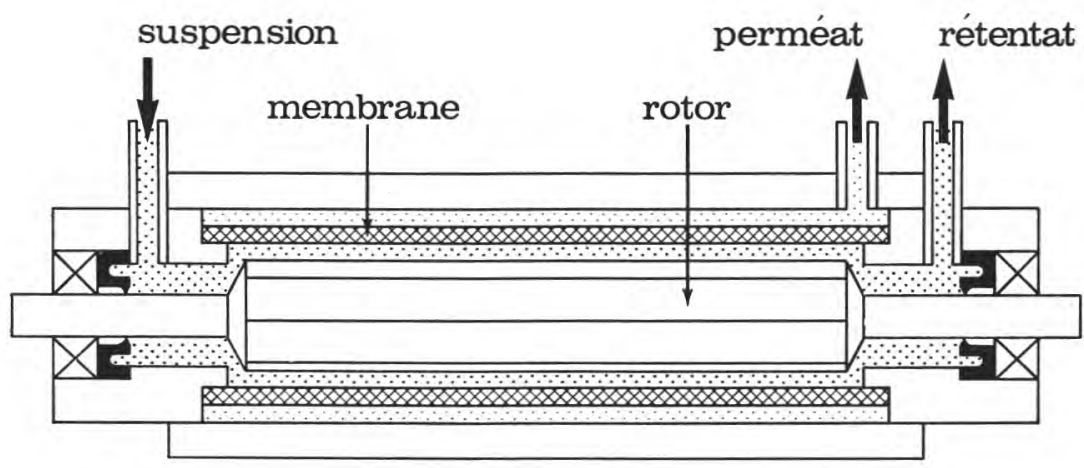

fig. 2

Cartouche de filtration. - Filtration module.

La cartouche est entourée d'un certain nombre d'organes modulaires afin de remplir les fonctions suivantes :

- alimentation de la cartouche et réglage de la pression de filtration ;

- réglage de la vitesse tangentielle;

- réglage du fractionnement perméat/rétentat;

- récupération des fluides;

- enregistrement du débit;

- régénération de la membrane par application séquentielle d'une pression inverse. 
Caractéristiques :

Pour la filtration :

Vitesse tangentielle de 0 à $4 \mathrm{~m} . \mathrm{s}^{-1}$;

- pression de filtration de 0 à 6 bars ;

- fractionnement volumique perméat/rétentat de 0 à 300 .

Pour le décolmatage :

— volume refoulé de 1 à $45 \mathrm{~cm}^{3}$;

- pression de refoulement de 0 à 6 bars;

- période de décolmatage de 1 à $45 \mathrm{~s}$.

Avantages de l'appareillage à mandrin rotatif. La pression de filtration et la vitesse tangentielle sont réglables indépendamment. Pour un taux de fractionnement donné la mise en équilibre est rapide. Le volume de fluide à traiter par essai n'est pas important en raison de l'absence de boucle de recirculation.

\section{ETUDE DE LA FILTRATION}

\section{Modélisation}

Les paramètres influents sur la filtration sont très nombreux. Ils sont liés à la nature de la membrane (structure, épaisseur, porosité, mouillabilité....), à celle de la suspension (particules déformables ou non, viscosité, charge, présence de gaz dissous...) et aux conditions de filtration (pression, vitesse, température, coefficient de fractionnement...). Il est connu que la filtration tangentielle permet de ralentir, mais non d'éviter le colmatage des membranes; il convient donc d'étudier ce dernier pour en tirer des indications quant à la manière d'effectuer le décolmatage : refoulement d'un certain volume de perméat sous une certaine pression et à une cadence donnée. Le modèle étudié prend en compte des particules de taille voisine à celle des pores. Elles pénètrent dans la membrane où elles sont retenues par des forces de frottement. Le flux inverse de perméat doit vaincre ces forces pour régénérer la membrane.

Considérons une certaine section de membrane filtrant une suspension sous une pression différentielle $\Delta \mathrm{P} 1$. Les particules vont obstruer progressivement les pores de la première couche. Soit $\mathrm{x}$ le taux de pores bouchés (fig. 3). La perte de charge dans la première couche crôit de $\Delta \mathrm{P} 0=\Delta \mathrm{P} 1 /(\mathrm{n}+1)$ pour $\mathrm{x}=0$ à $\Delta \mathrm{Px}$ pour un état $\mathrm{x}$ de colmatage. Dans l'une quelconque des $\mathrm{n}$ couches sousjacentes, dont les pores sont libres, intercommunicants et traversés par le même fluide, le débit de la valeur initiale Do $=$ Cte $(\Delta \mathrm{P} 1-\Delta \mathrm{Po}) / \mathrm{n}$ à $\mathrm{Dx}=\mathrm{Cte}(\Delta \mathrm{P} 1-\Delta \mathrm{Px}) / \mathrm{n}$. 


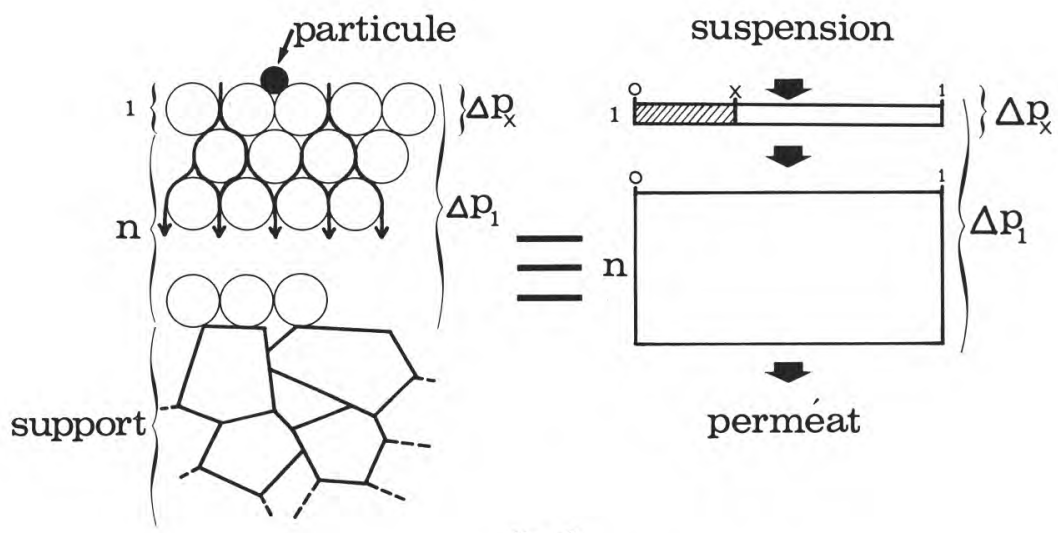

fig. 3

Représentation du modèle de filtration.

Schematic illustration of the filtration model.

L'expression du débit relatif ne dépend que de la perte de charge relative $\Delta \mathrm{Px} / \Delta \mathrm{P} 1$ dans la première couche et du nombre de couches n, soit :

$$
\mathrm{Dx}=\mathrm{Do}\left(1+\frac{1}{\mathrm{n}}\right)\left(1-\frac{\Delta \mathrm{Px}}{\Delta \mathrm{P} 1}\right)
$$

Par ailleurs, les débits dans la première couche et dans l'une quelconque des couches sous-jacentes sont égaux, donc :

$$
\Delta \mathrm{Px}(1-\mathrm{x})=(\Delta \mathrm{P} 1-\Delta \mathrm{Px}) / \mathrm{n}
$$

La perte de charge due à la première couche peut être exprimée en fonction du taux de colmatage $\mathrm{x}$ :

$$
\Delta \mathbf{P}_{\mathrm{x}}=\frac{\Delta \mathbf{P}_{1}}{\mathrm{n}(1-\mathrm{x})+1}
$$

Cette expression permet de relier le débit au taux de colmatage :

$$
\mathrm{D}_{\mathrm{x}}=\mathrm{D}_{\mathrm{o}}\left[1-\frac{\mathrm{x}}{\mathrm{n}(1-\mathrm{x})+1}\right]
$$

Le nombre de pores bouchés par unités de temps est fonction de la concentration $\mathrm{C}$ en particules de la suspension et du nombre total $\mathrm{N}$ des pores de la première couche de la membrane :

$$
\frac{d_{x}}{d_{t}}=\frac{D_{x} \cdot C}{N}
$$


Par intégration en tenant compte des conditions initiales $\mathrm{x}=0$, $\mathrm{D}_{\mathrm{x}}=\mathrm{D}_{\mathrm{o}}$ pour $\mathrm{t}=0$, il vient :

$$
t=\frac{N}{(n+1) C D_{0}}\left[n x-\log _{e}(1-x)\right]
$$

Cette expression est représentée par la courbe 1 de la figure 4 . L'exploitation des courbes résultant des relations (3) et (4) permet $\mathrm{D}_{\mathrm{t}}$

de tracer l'évolution du débit relatif - en fonction du temps (courbe 2 D。

de la figure 4). On constate que cette variation de débit n'est pas représentative du taux de colmatage jusqu'à $\mathrm{x}=0,8$ environ.

Les contraintes subies par les particules colmatantes sont dues à la perte de charge $\Delta \mathrm{Px}$ dont la valeur locale dépend étroitement de la distribution des pores libres alors que sa valeur moyenne s'exprime simplement. Lors d'une opération de décolmatage par flux inverse de perméat, la pression différentielle $\Delta \mathrm{P}_{2}$ ne peut physiquement s'établir instantanément et comme les contraintes subies par les particules présentent une dispersion due à celle de $\Delta \mathrm{Px}$, tous les pores ne se débouchent pas simultanément.

La pression $\Delta \mathrm{Px}$, s'exerçant sur les particules encore retenues, est fonction du taux de rétention $\mathrm{x}^{\prime}$ :

$$
\Delta \mathrm{P}_{\mathrm{x}},=\frac{\Delta \mathrm{P}_{2}}{\mathrm{n}\left(1-\mathrm{x}^{\prime}\right)+1}
$$

Les particules étant soumises, au cours de la filtration sous une pression différentiele $\Delta \mathrm{P}_{1}$, à une pression $\Delta \mathrm{P}_{\mathrm{x}}$ donnée par la relation (2), elles ne pourraient être chassées que si la pression $\Delta \mathbf{P}_{\mathrm{x}}$, s'exerçant en sens inverse est supérieure ou égale à $\Delta \mathrm{P}_{\mathrm{x}}$, soit $\Delta \mathrm{P}_{\mathrm{x}}, \geqslant \Delta \mathrm{P}_{\mathrm{x}}$.

Cette condition permet de calculer le taux de rétention $\mathrm{x}^{\prime}$ minimum que l'on peut atteindre à partir d'un état de colmatage $\mathrm{x}$ :

$$
\mathrm{x}^{\prime}=\frac{\Delta \mathrm{P}_{2}}{\Delta \mathrm{P}_{1}} \mathrm{x}+\frac{(\mathrm{n}+1)}{\mathrm{n}}\left(1-\frac{\Delta \mathrm{P}_{2}}{\Delta \mathrm{P}_{1}}\right)
$$

La régénération totale est obtenue pour $\mathrm{x}^{\prime}=0$ d'où la condition d'efficacité du décolmatage :

$$
\frac{\Delta \mathrm{P}_{2}}{\Delta \mathrm{P}_{1}}=\frac{\mathrm{n}+1}{\mathrm{n}(\mathrm{x}-1)+1}
$$

Remarquons que si le colmatage est total $(x=1)$ le décolmatage peut être encore effectué mais avec une pression dont la valeur est liée à la structure de la membrane : $\Delta \mathrm{P}_{2}=\Delta \mathrm{P}_{1}(\mathrm{n}+1)$. 
Le rapprochement des relations (4) et (6) permet de tracer l'évolution du rapport des pressions de filtration et de décolmatage en fonction du temps (courbe 3 de la figure 4).

$\mathrm{n}=\mathbf{3 5}$

$\mathrm{D}_{\mathrm{o}}=1 \mathrm{~m}^{3} \cdot \mathrm{m}^{-2} \cdot \mathrm{h}^{-1}$

$10^{8}$ pores par $\mathrm{cm}^{2}$

$10^{8}$ particules par $\mathrm{cm}^{3}$

1 : taux de colmatage $\mathrm{x}$

2 : débit relatif $D_{t} / D_{0}$

3 : condition d'efficacité du décolmatage $\Delta \mathrm{P}_{2} / \Delta \mathrm{P}_{1}$

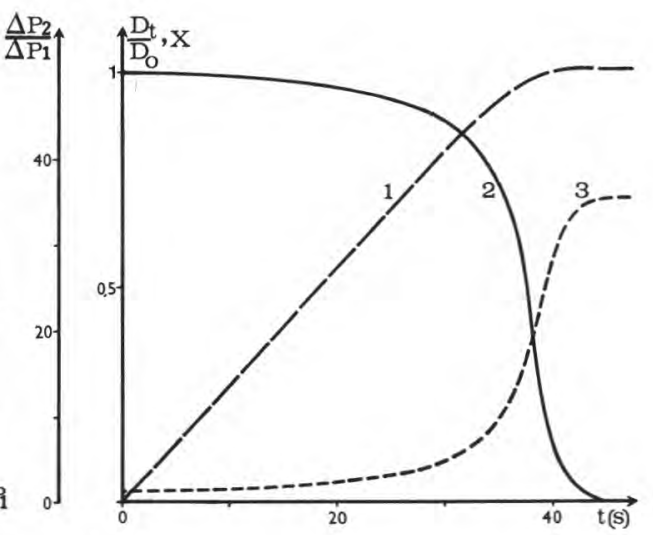

fig. 4

Courbes théoriques de l'évolution du taux de colmatage du débit de perméat et des conditions de décolmatage en fonction du temps.

Calculated curves for time dependence of clogging ratio, permeate flux and backflush conditions.

Ce modèle, malgré les hypothèses simplificatrices retenues, permet de définir de grandes lignes de conduite pour la filtration tangentielle avec décolmatage au moyen des résultats suivants :

- le débit relatif s'exprime simplement en fonction du nombre de couches formant la membrane et de la perte de charge dans la première couche au contact de la suspension;

- le taux de colmatage ne peut être représenté valablement par la variation relative du débit;

- le modèle du décolmatage montre que plus sa période est grande, plus la pression de refoulement doit être forte pour être efficace ;

- en présence de décolmatage, la vitesse tangentielle devient un paramètre secondaire car elle ne joue que sur la limitation de l'épaisseur du gâteau qui se reforme entre chaque décolmatage.

Ce modèle peut être étendu au cas de suspensions dont les particules ont un spectre de granulométrie étendue, qu'elles soient déformables ou non (Galaj et Gillot, 1983). 


\section{Etude expérimentale}

La recherche des conditions de stabilité de débit a été effectuée sur de l'eau de ville et du vin; dans de telles suspensions, les particules obstruant complètement les pores peuvent être des bactéries ou des micelles colloïdales.

Eau de ville (Site de Marcoussis). Les essais ont été effectués avec les conditions expérimentales décrites dans le tableau 2 avec une membrane de $0,2 \mu \mathrm{m}$. Les résultats de filtration sont présentés sur les courbes 1 à 7 de la figure 5 . La courbe 1 montre que le débit chute rapidement; après $30 \mathrm{~min}$ de filtration et sans changer de membrane, le fractionnement des fluides est ajusté à 25 et le décolmatage est déclenché pour chaque fraction de $40 \mathrm{~cm}^{3}$ de perméat récupéré; le débit n'évolue plus (courbe 2). En appliquant ces nouvelles conditions à un tube neuf et en portant $\Delta \mathrm{P}_{2}$ à 6 bars, le débit
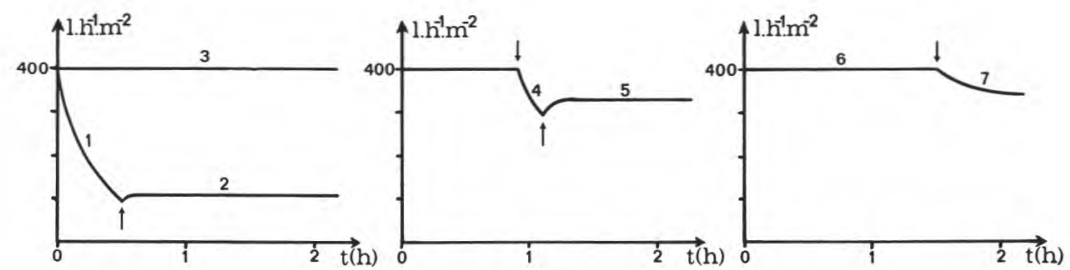

fig. 5

Filtration de l'eau de ville. - Filtration of tap-water.

TABLEAU 2 - TABLE 2

Conditions expérimentales pour la filtration d'eau de ville

Filtration of tap-water.

\begin{tabular}{l|rrrrrrr}
\hline Pression de filtration $\mathrm{P}_{1}$ (bar) & 0,5 & 0,5 & 0,5 & 0,5 & 0,5 & 0,5 & 0,5 \\
Vitesse tangentielle (m.s $\left.{ }^{-1}\right)$ & 1 & 1 & 1 & 1 & 1 & $1-2$ & 0,2 \\
Coefficient de fractionnement & 50 & 25 & 25 & 25 & 25 & 25 & 25 \\
Pression inverse $\mathrm{P}_{2}$ (bar) & 3 & 3 & 6 & 0 & 6 & 6 & 6 \\
Volume refoulé (cm $\left.{ }^{3}\right)$ & 10 & 10 & 10 & 0 & 10 & 10 & 10 \\
$\begin{array}{l}\text { Volume de perméat récupéré } \\
\left.\text { entre deux décolmatages (cm }{ }^{3}\right)\end{array}$ & 80 & 40 & 40 & - & 40 & 40 & 40 \\
Numéro de la courbe & 1 & 2 & 3 & 4 & 5 & 6 & 7 \\
\hline
\end{tabular}


conserve sa valeur initiale (courbe 3). Si l'opération de décolmatage est suspendue (pendant $10 \mathrm{~min}$ environ), le débit chute (courbe 4), le rétablissement du décolmatage conduit à un palier du débit (courbe 5) mais ne permet pas de retrouver le débit initial. Lorsque la vitesse tangentielle varie de 1 à $2 \mathrm{~m} . \mathrm{s}^{-1}$, le débit demeure constant (courbe 6). Par contre pour $0,2 \mathrm{~m} . \mathrm{s}^{-1}$ on observe une baisse du débit (courbe 7).

Ces différentes observations montrent que pour une pression de filtration et une vitesse tangentielle données, le débit peut être stable en conservant sa valeur d'arigine (courte période de décolmatage) ou bien variable et décroissant (longue période de décolmatage). Ce double comportement est révélateur d'une valeur critique de colmatage qu'il convient de ne pas dépasser.

Vin brut. Les vins bruts contiennent, outre des levures et des bactéries, des substances sous forme colloïdale. La suspension expérimentée est un vin blanc brut Mâcon 1981 prélevé en fin de fermentation. Un tel vin colmate totalement et rapidement des membranes $0,2 \mu \mathrm{m}$ en filtration frontale.

$\mathrm{Z}$

La figure 6 représente les variations de débit obtenu avec les conditions opératoires suivantes : $\Delta \mathrm{P}_{1}=0,5$ bar, $\Delta \mathrm{P}_{2}=6$ bars, fractionnement perméat $/$ rétentat $=20$, volume refoulé $=10 \mathrm{~cm}^{3}$, décolmatage pour chaque fraction de $20 \mathrm{~cm}^{3}$ récupérée. Les parties a, $\mathrm{b}$ et $\mathrm{c}$ de la courbe correspondent respectivement à des vitesses tangentielles de 1,2 et $0,1 \mathrm{~m} \cdot \mathrm{s}^{-1}$. Une légère décroissance de débit est observée au cours de la première heure, puis le débit se stabilise. L'accroissement de vitesse de 1 à $2 \mathrm{~m} . \mathrm{s}^{-1}$ n'augmente que légèrement

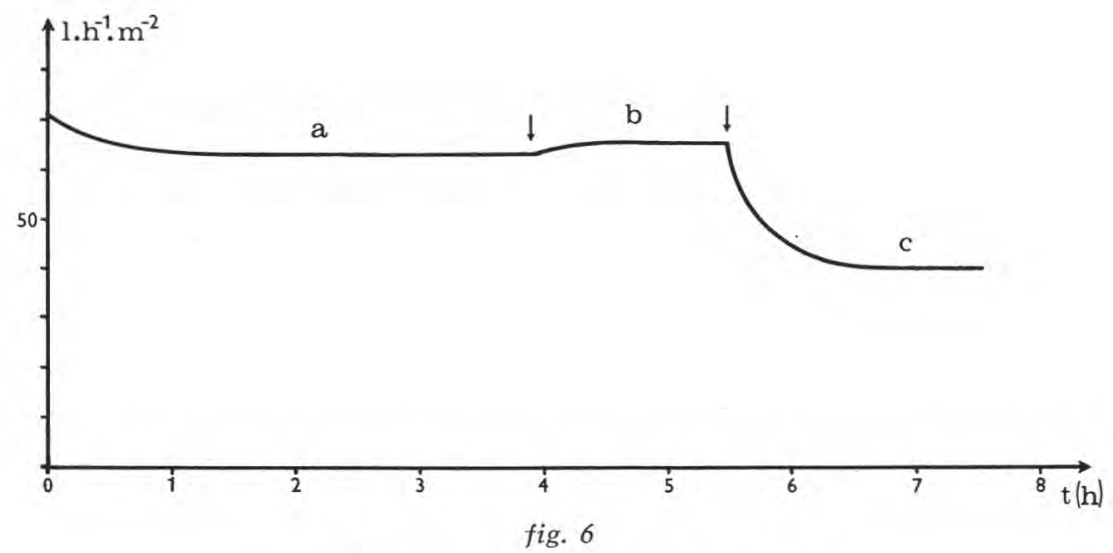

Filtration d'un vin brut en fin de fermentation.

Crude-wine filtration. 
le débit; par contre pour une vitesse bien plus faible, $0,1 \mathrm{~m} \cdot \mathrm{s}^{-1}$, le débit chute rapidement pour se stabiliser à nouveau. Bien que la vitesse paraisse n'avoir que peu d'influence sur le débit, elle doit être supérieure à un seuil ; en effet, si le gâteau décollé par l'opération de décolmatage n'est pas suffisamment entraîné, il se replaque presque aussitôt sur la membrane sans être désagrégé.

Quelques essais effectués sur des vins peu chargés conduisent à des débits nettement supérieurs, de l'ordre de $3001 . \mathrm{h}^{-1} \cdot \mathrm{m}^{-2}$ pour 0,5 bar. Une décroissance du débit en fonction du temps est cependant observée. Elle serait due au dégazage du gaz carbonique qui, s'il se produit dans les pores, conduit d'une part à leur obstruction et d'autre part rend inefficace l'action du décolmatage par effet d'amortissement.

\section{CONCLUSION}

L'objectif de toute filtration est d'arrêter les particules supérieures ou égales aux dimensions des pores de la membrane tout en conservant une bonne perméabilité du filtre. La filtration tangentielle avec décolmatage semble pouvoir atteindre cet objectif si l'on suit les indications suivantes : membrane à faible nombre de couches, pression de filtration basse et décolmatage fréquent sous forte pression. Ce type de filtration correspond à un fonctionnement intermédiaire enter les modes frontal et tangentiel. L'intérêt de cette technique est de réduire les coûts de filtration par la diminution des opérations de maintenance et par l'usage de vitesses d'entraînement plus faibles.

\section{Bibliographie}

Belfort (G.), Baltutis (T. F.), BlafF (W.F.) (1980). - Automated hollow fiber ultrafiltration: Pyrogen removal and phage recovery from water. In " Ultrafiltration membranes and applications » edited by A. R. Cooper, Plenum Press, New York.

Blatt (W. F.), Dravid (A.). Michaels (A. S.), Nelson (L.) (1970), - Solute polarization and cake formation in membrane ultrafiltration. Causes consequences and control techniques. In "Membrane science and technology » edited by J.E. Flinn, Plenum Press, New York.

Galaj (S.) Gillot (J.) (1983). - Etude de la microfiltration tangentielle avec décolmatage sur membranes céramiques. Compte rendu de fin d'étude D.G.R.S.T., $n^{\circ}$ 81.C.1278.

Howell (J. A.), Velicangil (O.) (1980). - Protein ultrafiltration: theory of membrane fouling and its treatment with immobilized proteases. In "Ultrafiltration membranes and applications" edited by A. R. Cooper, Plenum Press, New York.

Lopez-LeIva (M.) (1980). - Prediction of permeate fluxes in UF/RO systems. In "Ultrafiltration membranes and applications" edited by A. R. Cooper, Plenum Press, New York.

Porter (M. C.) (1972). - Ind. Eng. Chem. Prod. Res. Develop., 11 (3), 234. 